\title{
UCRL-TR-227253
}

LAWRENCE LIVERMORE N A T IO N A L LABORATORY
Sister Lab Program Prospective Partner Nuclear Profile: Vietnam

Mo Bissani, Sean Tyson

January 12, 2007 
This document was prepared as an account of work sponsored by an agency of the United States Government. Neither the United States Government nor the University of California nor any of their employees, makes any warranty, express or implied, or assumes any legal liability or responsibility for the accuracy, completeness, or usefulness of any information, apparatus, product, or process disclosed, or represents that its use would not infringe privately owned rights. Reference herein to any specific commercial product, process, or service by trade name, trademark, manufacturer, or otherwise, does not necessarily constitute or imply its endorsement, recommendation, or favoring by the United States Government or the University of California. The views and opinions of authors expressed herein do not necessarily state or reflect those of the United States Government or the University of California, and shall not be used for advertising or product endorsement purposes.

This work was performed under the auspices of the U.S. Department of Energy by University of California, Lawrence Livermore National Laboratory under Contract W-7405-Eng-48. 


\section{Sister Lab Program \\ Prospective Partner Nuclear Profile: Vietnam}

[28 September 2006]

\section{Background}

Vietnam's nuclear program began in the 1960s with the installation at Dalat of a 250 kW TRIGA Mk-II research reactor under the U.S. Atoms for Peace Program. The reactor was shut down and its core removed only a few years later, and the nuclear research program was suspended until after the end of the civil war in the late 1970s.

The Soviet Union assisted Vietnam in restoring the Dalat reactor to an operational status in 1984, trained a cadre of scientific and technical staff in its operation, and contributed to the development of nuclear science for the medical and agricultural sectors.

In the agricultural area in particular, Vietnamese experts have been very successful in developing mutant strains of rice, and continue to work

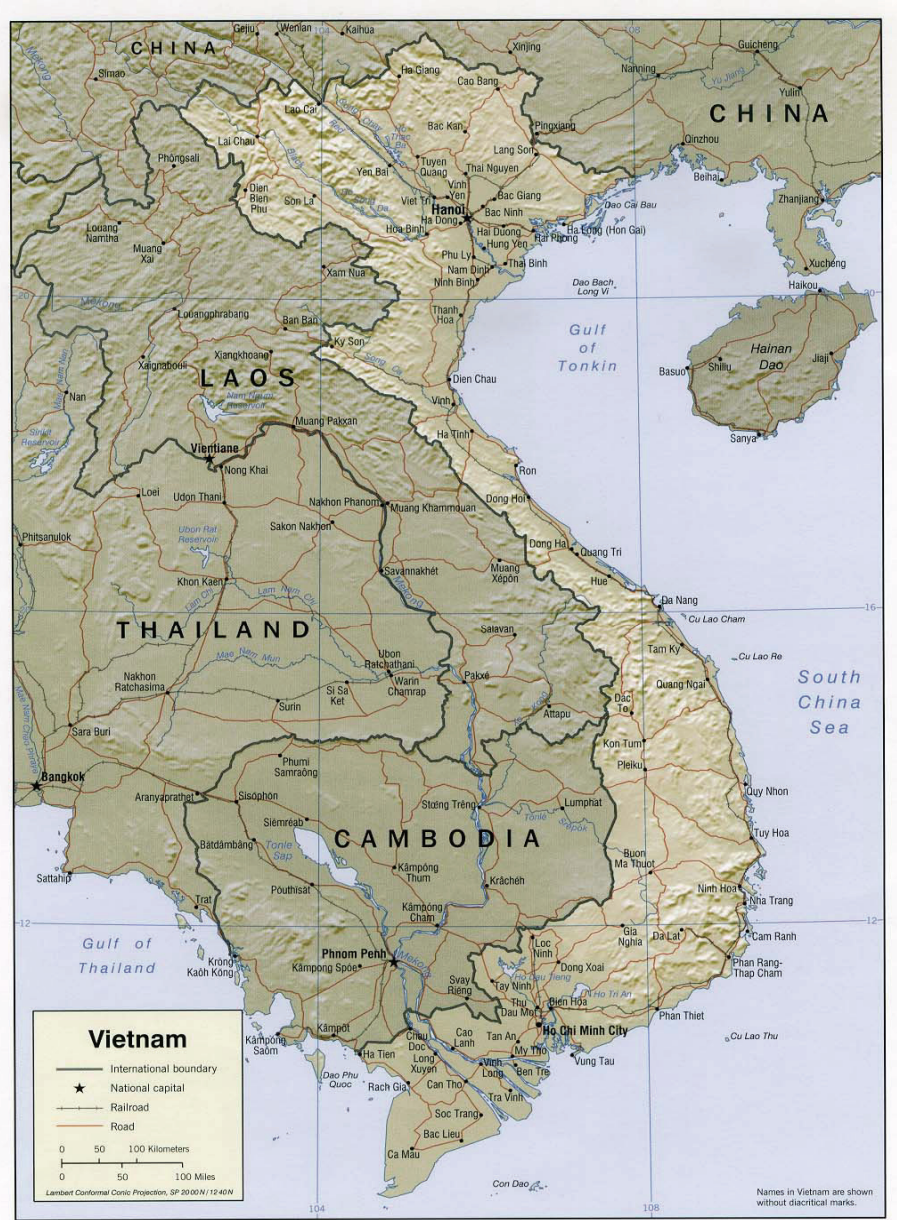

Source: University of Texas Library,

http://www.lib.utexas.edu/maps/middle_east_and_asia/vietnam_rel01.jpgVietnam with the IAEA to yield strains that have a shorter growing period, increased resistance to disease, and other desirable characteristics. ${ }^{1}$ Rice has always been the main crop in Vietnam, but technical cooperation with the IAEA and other states has enabled the country to become one of the top rice producers in the world, exporting much of its annual crop to over two dozen countries annually. ${ }^{2}$

More recently, Vietnam's government has shown increasing interest in developing a civil nuclear program to supplement its fossil fuel and other energy resources. Projections from a variety of open sources, ranging from the IAEA, the U.S. Department of Energy's Energy Information Administration (EIA), the Vietnamese government, energy 
corporations $^{3}$, and think tanks all predict a massive increase in energy consumptionespecially electricity-within Vietnam and the region as a whole.

This growth in consumption will require a corresponding increase in energy production, which in Vietnam is currently satisfied mainly by fossil fuels (coal) and renewable energy (hydropower and biomass); Vietnam has a refining capacity of about 800 barrels/day. Most of its crude oil is exported to generate export income, and is not used to generate electricity. ${ }^{4}$ Although Vietnam is able to meet most of its needs through its own resources, it consumes more electricity than it produces (approximately six billion $\mathrm{KWh} / \mathrm{a}){ }^{5}$ Open sources indicate that increasing exports of manufactured goods and a corresponding growth in the industrial sector $(16 \% \text { since } 2003)^{6}$ will lead to a greater energy shortfall beyond the next decade - between 35 and 65 billion KWh/year after $2020{ }^{78}$ As a signatory to the Kyoto Treaty and other regional environmental accords, and seeking an alternative to its stretched hydroelectric resources, Vietnam is looking for a means to increase energy production while minimizing emissions of greenhouse gases. ${ }^{9}$

\section{Technical Capabilities and Facilities}

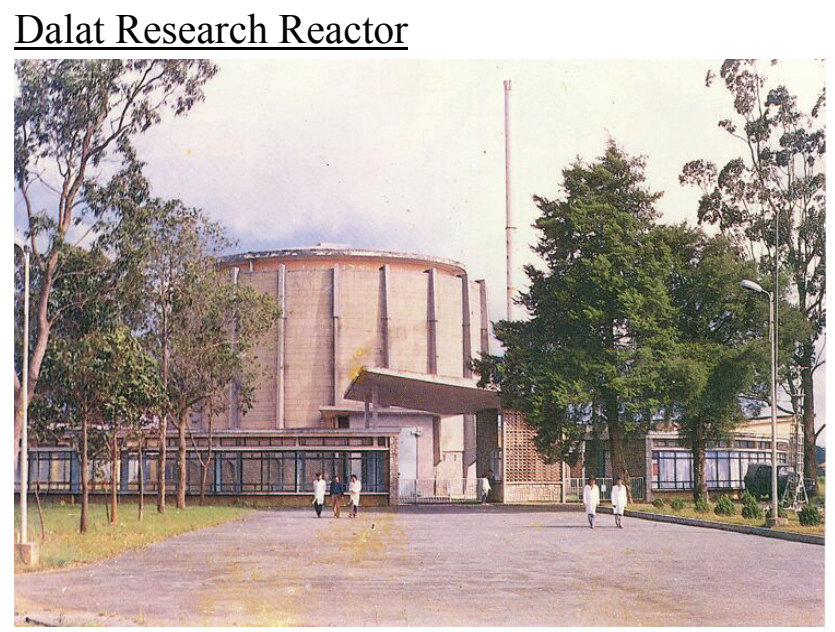

Vietnam's only research reactor is located at the Nuclear Research Institute (NRI) in Dalat. The reactor was originally built in the early 1960 s as a $250 \mathrm{~kW}$ TRIGA Mk-II pool-type research reactor. The reactor was shut down during the country's civil war in the 1960s and 1970s, and the fuel was removed to the United States in April 1975. In 1984 the reactor was restored to operating condition with the assistance of the Soviet Union, which installed a new core within the existing reactor tank. The restored $500 \mathrm{~kW}$ reactor utilizes WWR-M2 fuel assemblies enriched to $36 \%,{ }^{10}$ and has been primarily engaged in radioisotope production for the medical, agricultural, and industrial sectors, and to conduct research in these and environmentally-related areas. ${ }^{11}$ The age of the reactor and limitations on neutron flux and power level are beginning to restrict its ability to support increasing user demands; current plans by the NRI are to replace the reactor with a new high power research reactor before $2020 . .^{12}$

\section{Radiotherapy Centers}

Vietnam Agency for Radiation and Nuclear Safety and Control (VARANSAC) officials informed U.S. Department of Energy (DOE) officials that there are currently 13 Co-60 teletherapy units in use throughout Vietnam. Although DOE has little information on these units, it is known that three operate in Hanoi, several are located in Ho Chi Minh City and the remainder spread about the country. Three disused sources are stored in a warehouse in Ho Chi Minh City, in a purportedly secure facility. All of the sources are 
old and nearing their end of operational life. As the units were supplied to Vietnam without a source return policy, the country will be required to store them until they are no longer a hazard. ${ }^{13}$

$\underline{\text { Irradiation Facilities }}$

VARANSAC representatives informed U.S. DOE officials that there are five irradiator facilities in use and one in storage in Vietnam. One of the facilities is located at the Vietnam Institute for Nuclear Science and Technique in Hanoi, and houses a unit that has approximately 25,000 Curies of activity. The facility is used to sterilize medical supplies and irradiate food. The Vietnam Atomic Energy Commission (VAEC), the facility operator, has plans to implement several upgrades to the unit by 2006, which will increase it to 300,000 Curies. At least two irradiators are located in Ho Chi Minh City, one of which had approximately 400,000 Curies in 1999. Little is known of their current status. $^{14}$

Waste Repository

Vietnam does not currently operate a national waste repository, nor does it have a location to securely store the Co-60 sources approaching their end of operational life. U.S. DOE experts have recommended a facility be designed and constructed to securely store these sources in the future. ${ }^{15}$

Educational Institutions ${ }^{16}$

Hanoi National University (HNU) - Department of Nuclear Physics, awards 30 degrees a year to students in nuclear physics.

Hanoi University of Technology (HUT) - Department of Nuclear Engineering and Environmental Physics, awards about 10 degrees per year in engineering. Dalat University - 10 degrees a year to graduate students in nuclear science. VAEC - Offers graduate- (Masters degree) and doctoral-level courses in mathematics, programming, physics, and radiation measurement and protection.

NRI - Training Center, supported by IAEA and BARC financial grants, offers radiation safety center, training in radiation protection and nuclear safety, nuclear technology and energy.

INST Joint Training Center on Radiation Protection - equipped by JAERI, offers courses in radiation protection and measurement, as well as instructor training.

EBP Activities - Offers national training in the direction of education and training, legislation, safety analysis, assessment and regulatory supervision for the Dalat RR.

\section{Nuclear Power}

In order to develop the energy infrastructure necessary to continued growth, Vietnam's government is examining the potential contribution of nuclear power to its economy. In addition to signing technical cooperation agreements in nuclear technology with a number of states, it has discussed construction of nuclear power plants with several countries, including Russia, ${ }^{17}$ France, the United States, Canada, and Japan. ${ }^{18}$ Japan has also offered to train scientific and technical personnel necessary to support operation of the anticipated nuclear power plant; ${ }^{19}$ on 25 August 2006, Toshiba Corporation, Hanoi 
University of Technology and VAEC signed an agreement for Toshiba to hold annual training at HUT's Department of Nuclear Engineering and Environmental Physics of the Institute of Engineering Physics for 25 graduate and postgraduate students. ${ }^{20}$

A number of officials from the Vietnam Atomic Energy Institute (VAEI) and the NRI in Dalat have predicted that Vietnam would build its first nuclear power plant between 2010 and 2020 in order to address an anticipated shortfall in electric generating capacity within the same period. The Vice Director of VAEI stated in October 2005 that the plant would possess a $2-4 \mathrm{GWe}$ generating capacity, and that a pre-feasibility study was already underway. ${ }^{21}$

\section{Nuclear Institutions}

Vietnamese nuclear organizations operate within the Ministry of Science and Technology, as shown below. Note that VARANSAC is not represented in this diagram, but also operates under MOST: ${ }^{22}$

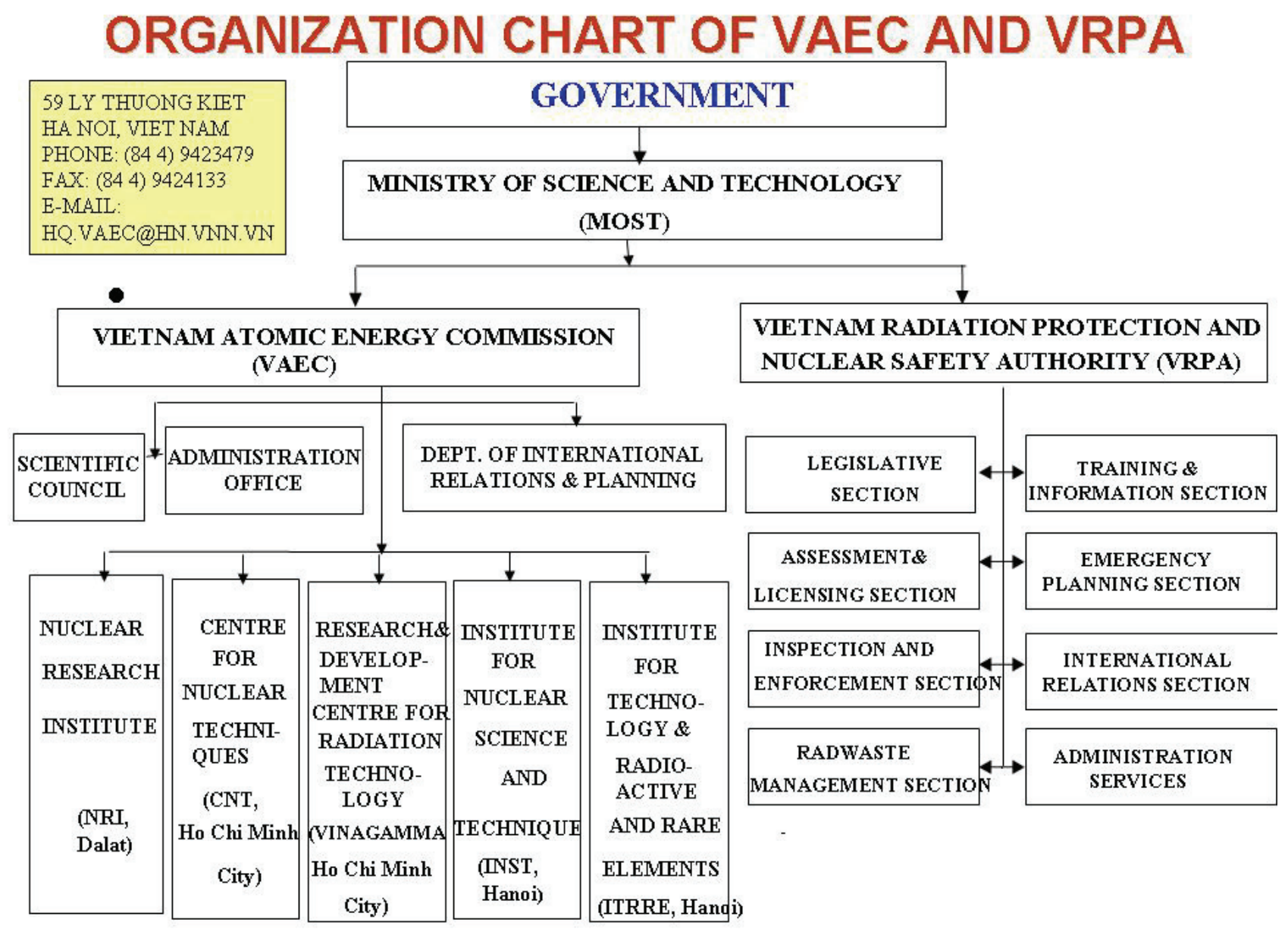

\subsection{Vietnam Atomic Energy Commission (VAEC)}


VAEC's main function is to perform research and development in the field of atomic energy. VAEC is headed by a Chairman, currently Dr. Tran Huu Phat; the Vice Chairman is Dr. Bui Van Tuan. ${ }^{23}$

\subsection{Vietnam Agency for Radiation and Nuclear Safety and Control (VARANSAC) ${ }^{\mathbf{2 4}}$} VARANSAC is a regulatory body under MOST with the duty of assisting the Minister in the state management of radiation and nuclear safety, and control. The organization is led by a Director General, currently Dr. Ngo Dang Nhan; the Deputy Director General is Dr. Dang Thanh Luong. Beneath the Director General's office are five departments, including the Departments of:

- Technical Assistance for Radiation and Nuclear Safety

- International Cooperation, Legislation and Information

- Inspection, Control and Emergency Response

- Registration and Licensing

- Administration and Planning

VARANSAC's main functions are as follows: ${ }^{25}$

1. To organize and participate in the formation of legislative documents, code of practice, procedures and regulations for radiation and nuclear safety and control;

2. To participate in the formation of standards on radiation and nuclear safety, specific regulations and policies for those who work directly with radiation;

3. To contribute to and submit policies, development orientations, priorities, programs, annual and 5-year plans on radiation and nuclear safety and control to the Minister; to organize and implement approved plans;

4. To organize and implement the notification, registration, license, renewal, amendment and withdrawal of licenses for radiation and nuclear establishments, radioactive sources, radiation personnel, and works relating to radiation and nuclear issues;

5. To organize the assessment of sites, designs, construction, and justifications for ensuring the radiation and nuclear safety and security of radiation and nuclear establishments;

6. To guide and direct the Local Departments of Science and Technology on radiation and nuclear safety and control; to coordinate with Ministries and Branches to perform the State management on the radiation and nuclear safety and control under the MOST's direction;

7. To conduct regulatory inspections on radiation and nuclear safety according to law;

8. To resolve complaints, denunciations; to deal with violations of regulations on radiation safety and control according to law;

9. To provide State management of radioactive waste;

10. To organize radiation environmental monitoring, to develop emergency response and handling for radiation and nuclear incidents;

11. To control radiation doses and assess the safety of occupational, public and medical exposure;

12. To organize safeguards activities;

13. To establish a record system of data, information on radiation and nuclear safety; 
14. To organize research for applying scientific and technological advances in the field of radiation safety and control;

15. To co-organize training course, propaganda and dissemination programs on legislation, radiation and nuclear safety, and safety culture

\subsection{Radiation and Nuclear Safety and Controls ${ }^{26}$}

The Ministry of Science and Technology (MOST) oversees the operations of 64 Departments of Science and Technology which are spread throughout the country.

\subsubsection{Overview of National Activities}

The action plan for the Radiation and Nuclear Safety and Controls is focused on several main tasks, including:

1. Developing and improving the regulatory system

2. Establishing Occupational Exposure Controls

3. Establishing Medical Exposure Controls

4. Establishing the Public Exposure Controls

5. Safe Management of Radioactive Waste; Safety and Security for disused Sources and Radioactive materials

6. Establishing the Emergency Response and Preparedness

7. Developing calibration facilities

8. Manpower development and Public Information

9. License and Inspections for the Dalat Research Reactor

\subsubsection{The National Legal Framework Concerning Radiation Protection and Control}

1. Basic Atomic Law, Ordinance on Radiation Control

2. Decree 50/1998/ND-CP on Implementation of Ordinance on Radiation Safety and Control

3. Decree 19/2001/ND-CP on Punishment of Violation of Ordinance on Radiation Safety and Control

4. Circulars on radiation protection, management of radioactive waste, transport of radioactive material, fees and fares, and licensing and inspections. 


\section{Bilateral and Multilateral Nuclear Technical Cooperation}

Vietnam has engaged in considerable work both with the IAEA (as indicated below), bilaterally, and on a regional/international basis in developing agricultural, medical and industrial uses for nuclear technology. Below is a chronological summary of projects that are active, complete, or cancelled to date. ${ }^{27}$ In general, the tables below indicate that Vietnamese scientific and technical personnel have been involved in a very broad range of projects building their expertise and capabilities in many areas utilizing nuclear technology. Without more detail, it is difficult to state the level of expertise of this community, but there appears to be a solid basis for the long term development of a more advanced national nuclear energy sector, as well as expanded programs supporting agriculture, and medicine and industry.

A brief review of national project titles indicates that in addition to the medical and agricultural fields, Vietnam has been involved in a broad range of activities involving nuclear technology, including personnel health and safety, law, reactor operations, materials handling/metallurgy, and education. Its personnel have also upgraded instrumentation and control equipment for its Dalat research reactor and have trained in processing uranium ore.

Within the region, Vietnamese experts have participated in training on nuclear regulatory issues, nuclear health-related issues, radioactive waste management, maintenance of national nuclear institutions, numerous medical and agricultural courses employing nuclear technology, and the operation and maintenance of nuclear research and power facilities and infrastructures.

\subsection{National Projects:}

$\begin{array}{cl}\begin{array}{c}\text { Active National } \\ \text { Project } \\ \text { Number }\end{array} & \begin{array}{l}\text { Projects (14) } \\ \text { Title }\end{array} \\ \text { VIE0008 } & \begin{array}{l}\text { Human Resource Development and Nuclear } \\ \text { Technology Support }\end{array} \\ \text { VIE5014 } & \begin{array}{l}\text { Rice Mutant Varieties for Saline Land, Phase II } \\ \text { VIE6021 }\end{array} \\ & \begin{array}{l}\text { Application of Accelerator Technique for Medical } \\ \text { Treatment }\end{array}\end{array}$

\begin{tabular}{crl} 
Field & $\begin{array}{c}\text { 1st Year of } \\
\text { Approval }\end{array}$ & Country Officer \\
OA & 1995 & $\begin{array}{l}\text { Kamel, Reyad Sherif } \\
\text { (TCAP) }\end{array}$ \\
5C & 1999 & $\begin{array}{l}\text { Kamel, Reyad Sherif } \\
\text { (TCAP) } \\
\text { Kamel, Reyad Sherif } \\
\text { (TCAP) }\end{array}$ \\
\hline
\end{tabular}

Project Responsible
Officer
Kamel, Reyad Sherif
(TCAP)
Kamel, Reyad Sherif
(TCAP)
Kamel, Reyad Sherif
(TCAP)

Technical Officer

El Khangi, Fathi (TCAP)

Shu, Qingyao (NAFA)

Vatnitsky, Stanislav (NAHU) Rosenblatt, Eduardo (NAHU)
Total Budge 1202262 731725 351660 


$\begin{array}{ll}\text { VIE8014 } & \begin{array}{l}\text { Upgrading the Irradiation Facility at Hanoi Irradiation } \\ \text { Centre }\end{array} \\ \text { VIE8016 } & \begin{array}{l}\text { Isotope and Nuclear Techniques for Better } \\ \text { Management of Groundwater }\end{array} \\ \text { VIE9008 } & \begin{array}{l}\text { Establishment of a National Radiation Safety } \\ \text { Training Centre }\end{array} \\ \text { VIE0010 } & \begin{array}{l}\text { Technical Support for Training in Nuclear } \\ \text { Engineering at the Hanoi University of Technology }\end{array} \\ \text { VIE0011 } & \begin{array}{l}\text { Human Resource Development and Nuclear } \\ \text { Technology Support }\end{array} \\ \text { VIE4014 } & \begin{array}{l}\text { Modification of the Dalat Reactor Control System } \\ \text { VIE5015 }\end{array} \\ & \text { Enhancement of Quality and Yield of Rice Mutants } \\ & \text { Using Nuclear and Related Techniques, Phase II } \\ \text { VIE6023 } & \begin{array}{l}\text { Establishment of National Cyclotron Facilities and } \\ \text { Centres for Medical and Research }\end{array}\end{array}$

VIE8017

Upgrading the Irradiation Facility at Hanoi Irradiation Centre

VIE8018

Applying Electron Beam Technology for Processing Biomaterials

VIE9009
2001 Kamel, Reyad Sherif (TCAP)

2001 Kamel, Reyad Sherif (TCAP)

2001 Kamel, Reyad Sherif (TCAP)

2003 Kamel, Reyad Sherif (TCAP)

2003 Kamel, Reyad Sherif (TCAP)

2003 Kamel, Reyad Sherif (TCAP)

2005 Kamel, Reyad Sherif (TCAP)

2005 Kamel, Reyad Sherif (TCAP)

2005 Kamel, Reyad Sherif (TCAP)

2005 Kamel, Reyad Sherif (TCAP)

2005 Kamel, Reyad Sherif (TCAP)
Kamel, Reyad Sherif (TCAP)

Kamel, Reyad Sherif (TCAP)

Kamel, Reyad Sherif (TCAP)

Kamel, Reyad Sherif (TCAP)

Kamel, Reyad Sherif (TCAP)

Kamel, Reyad Sherif (TCAP)

Kamel, Reyad Sherif (TCAP)

Kamel, Reyad Sherif (TCAP) (NENP) Laurent (NAFA)

Kamel, Reyad Sherif

Kamel, Reyad Sherif (TCAP)

Kamel, Reyad Sherif Philip, George (NS) (TCAP)
Sampa, Maria Helena de O. (NAPC)

Kulkarni, Kshitij Mahadev (NAPC)

Sadagopan, Geetha (NSRW)

Einav, Isaac (NAPC) Glockler, Oszvald

Kamel, Reyad Sherif (TCAP)

Paranjpe, Shriniwas Krishnarao (NAPC) Ciuculescu, Cristina (NSNI)

Shu, Qingyao (NAFA) Lagoda, Pierre Jean

Izewska, Joanna (NAHU)

Haji-Saeid, Seyed Mohammad (NAPC) Bokulic, Tomislav (NAHU) Sampa, Maria Helena de O. (NAPC)

Haji-Saeid, Seyed Mohammad (NAPC)

Sampa, Maria Helena de O. (NAPC)

34300

283370

154666

213840

820570

243220

456692

310620

352650

88410

\section{Completed National Projects (77)}




\begin{tabular}{|c|c|}
\hline $\begin{array}{l}\text { Project } \\
\text { Number }\end{array}$ & Title \\
\hline VIE1002 & Radiation Dosimetry \\
\hline VIE5007 & Food Preservation \\
\hline VIE6007 & Radioisotopes in Medicine \\
\hline VIE6008 & Radioisotopes in Medicine \\
\hline VIE9002 & Personnel Monitoring \\
\hline VIE2002 & Trace Elements Analysis \\
\hline VIE6009 & Radioisotopes in Medicine \\
\hline VIE5009 & Food Irradiation \\
\hline VIE1003 & Nuclear Physics \\
\hline VIE1006 & Nuclear Physics (Hanoi University) \\
\hline VIE4002 & Nuclear Electronics Laboratory \\
\hline VIE6010 & Nuclear Medicine \\
\hline VIE1004 & Nuclear Physics Teaching \\
\hline VIE4004 & Nuclear Fuel for Research Reactor \\
\hline VIE9003 & Radiation Monitoring \\
\hline VIE5008 & Plant Mutation Breeding \\
\hline VIE5010 & Isotope Techniques in Soil-Plant Studies \\
\hline
\end{tabular}

\begin{tabular}{|c|c|c|}
\hline Field & $\begin{array}{l}\text { Completed } \\
\text { on }\end{array}$ & Country Officer \\
\hline $1 \mathrm{~K}$ & $1979-11-19$ & $\begin{array}{l}\text { Sarac, Abbas Ilhan } \\
\text { (SGOB) }\end{array}$ \\
\hline $5 \mathrm{H}$ & $1979-11-19$ & $\begin{array}{l}\text { Sarac, Abbas Ilhan } \\
\text { (SGOB) }\end{array}$ \\
\hline $6 B$ & $1979-11-19$ & $\begin{array}{l}\text { Sarac, Abbas Ilhan } \\
\text { (SGOB) }\end{array}$ \\
\hline $6 B$ & $1979-11-19$ & $\begin{array}{l}\text { Sarac, Abbas Ilhan } \\
\text { (SGOB) }\end{array}$ \\
\hline $\begin{array}{l}6 \mathrm{~F} \\
9 \mathrm{C}\end{array}$ & $1979-11-19$ & $\begin{array}{l}\text { Sarac, Abbas Ilhan } \\
\text { (SGOB) }\end{array}$ \\
\hline $2 \mathrm{C}$ & $1981-08-21$ & $\begin{array}{l}\text { Sarac, Abbas Ilhan } \\
\text { (SGOB) }\end{array}$ \\
\hline $6 \mathrm{~B}$ & $1982-11-23$ & $\begin{array}{l}\text { Sarac, Abbas Ilhan } \\
\text { (SGOB) }\end{array}$ \\
\hline $5 \mathrm{H}$ & 1983-01-07 & $\begin{array}{l}\text { Sarac, Abbas Ilhan } \\
\text { (SGOB) }\end{array}$ \\
\hline 1D & $1983-07-12$ & $\begin{array}{l}\text { Sarac, Abbas Ilhan } \\
\text { (SGOB) }\end{array}$ \\
\hline $\begin{array}{l}1 \mathrm{D} \\
1 \mathrm{~L}\end{array}$ & $1983-07-12$ & $\begin{array}{l}\text { Sarac, Abbas Ilhan } \\
\text { (SGOB) }\end{array}$ \\
\hline $4 G$ & $1983-08-22$ & $\begin{array}{l}\text { Sarac, Abbas Ilhan } \\
\text { (SGOB) }\end{array}$ \\
\hline $6 B$ & $1983-08-22$ & $\begin{array}{l}\text { Sarac, Abbas Ilhan } \\
\text { (SGOB) }\end{array}$ \\
\hline $1 \mathrm{D}$ & $1983-09-13$ & $\begin{array}{l}\text { Sarac, Abbas Ilhan } \\
\text { (SGOB) }\end{array}$ \\
\hline 4B & 1984-09-17 & $\begin{array}{l}\text { Sarac, Abbas llhan } \\
\text { (SGOB) }\end{array}$ \\
\hline $\begin{array}{l}9 \mathrm{~K} \\
9 \mathrm{C}\end{array}$ & $1984-10-19$ & $\begin{array}{l}\text { Sarac, Abbas Ilhan } \\
\text { (SGOB) }\end{array}$ \\
\hline $5 C$ & $1985-10-16$ & $\begin{array}{l}\text { Bautista, Eumelia R.B. } \\
\text { (SGCP) }\end{array}$ \\
\hline $5 B$ & $1985-10-16$ & $\begin{array}{l}\text { Bautista, Eumelia R.B. } \\
\text { (SGCP) }\end{array}$ \\
\hline
\end{tabular}

Project Responsible Technical Officer Officer

Total Budget

3558

24480

2225

0

Dolnicar, Joze (NAPC)

26753

Vavrejn, Bohuslav

(RILS)

Loaharanu, Paisan

(NAFA)

Dolnicar, Joze (NAPC)

Dolnicar, Joze (NAPC)

Dolnicar, Joze (NAPC)

Vavrejn, Bohuslav

(RILS)

Dolnicar, Joze (NAPC)

Dolnicar, Joze (NAPC)

Dolnicar, Joze (NAPC)

Maluszynski, Miroslav (NAFA)

Lamm, Carl Goeran

(NAFA)
36168

4868

68818

34047

88303

61676

114131

550658

117950

157648

3062 


\begin{tabular}{|c|c|c|c|c|}
\hline VIE8002 & Nuclear Techniques in Metallurgy & $\begin{array}{l}8 \mathrm{E} \\
1 \mathrm{G}\end{array}$ & $1985-10-16$ & $\begin{array}{l}\text { Bautista, Eumelia R.B. } \\
\text { (SGCP) }\end{array}$ \\
\hline VIE4005 & Nuclear Manpower Development & $\mathrm{OB}$ & $1986-11-10$ & $\begin{array}{l}\text { Bautista, Eumelia R.B. } \\
\text { (SGCP) }\end{array}$ \\
\hline VIE0003 & Nuclear Information Centre & $\begin{array}{l}\text { OF } \\
0 J\end{array}$ & 1989-09-25 & $\begin{array}{l}\text { Bautista, Eumelia R.B. } \\
\text { (SGCP) }\end{array}$ \\
\hline VIE1005 & Nuclear Physics (Dalat University) & $\begin{array}{l}1 \mathrm{D} \\
1 \mathrm{~L}\end{array}$ & 1989-09-25 & $\begin{array}{l}\text { Bautista, Eumelia R.B. } \\
\text { (SGCP) }\end{array}$ \\
\hline VIE1007 & Experimental Nuclear Physics & $\begin{array}{l}1 \mathrm{D} \\
1 \mathrm{~L}\end{array}$ & $1989-09-25$ & $\begin{array}{l}\text { Bautista, Eumelia R.B. } \\
\text { (SGCP) }\end{array}$ \\
\hline VIE2003 & Radiation Polymerization & $2 \mathrm{D}$ & $1989-09-25$ & $\begin{array}{l}\text { Bautista, Eumelia R.B. } \\
\text { (SGCP) }\end{array}$ \\
\hline VIE6013 & Nuclear Medicine (Dalat) & $6 \mathrm{~B}$ & $1989-09-25$ & Aslam, Javed (TCPA) \\
\hline VIE0004 & Preparatory Studies for a Nuclear Power Project & $O A$ & $1989-09-28$ & Aslam, Javed (TCPA) \\
\hline VIE6011 & Radioisotopes in Medicine & $6 G$ & $1989-09-28$ & $\begin{array}{l}\text { Bautista, Eumelia R.B. } \\
\text { (SGCP) }\end{array}$ \\
\hline VIE4006 & Computer Centre for Reactor Physics & $\begin{array}{l}1 F \\
4 \mathrm{D}\end{array}$ & $1990-12-28$ & Aslam, Javed (TCPA) \\
\hline VIE9005 & $\begin{array}{l}\text { Underwater Inspection of Dalat Nuclear Research } \\
\text { Reactor }\end{array}$ & $9 \mathrm{~F}$ & $1990-12-28$ & Aslam, Javed (TCPA) \\
\hline VIE6012 & Nuclear Medicine (Hue) & $6 \mathrm{~B}$ & $1991-01-15$ & Aslam, Javed (TCPA) \\
\hline VIE4003 & Nuclear Instrumentation & $4 G$ & $1991-12-31$ & Aslam, Javed (TCPA) \\
\hline VIE4007 & $\begin{array}{l}\text { Nuclear Instrumentation and Microcomputer } \\
\text { Applications }\end{array}$ & $4 G$ & $1991-12-31$ & Aslam, Javed (TCPA) \\
\hline VIE4008 & Nuclear Track Detectors & $4 G$ & $1991-12-31$ & Aslam, Javed (TCPA) \\
\hline VIE6016 & $\begin{array}{l}\text { TECHNETIUM-99m PRODUCTION AND QUALITY } \\
\text { CONTROL }\end{array}$ & $\begin{array}{l}6 G \\
2 F\end{array}$ & $1991-12-31$ & Aslam, Javed (TCPA) \\
\hline
\end{tabular}

Markovic, Vitomir M. (NAPC)

Ganatra, Ramanik D.

(NAHU)

Molina, Pablo (NENP) Calori, Fausto (NENP)

Ganatra, Ramanik D.

(NAHU)

Muranaka, Richard

George G. (NAPC)

Bernatowicz, Marek

Edmund (NENS)

Gopinathan Nair,

Gopalan (NAHU)

Dodwell (NAHU)

Dolnicar, Joze (NAPC)

Dolnicar, Joze (NAPC)

Ahmed, Jasim Uddin (NENS)

Vera Ruiz, Hernan Guido (NAPC) 
VIE0005

Nuclear Information Centre
VIE5011

Isotope Techniques in Soil Plant Studies

VIE8006

VIE8003

VIE5012

VIE9006

VIE0007

VIE8004

VIE1008

VIE4009

VIE4011
Nuclear Medicine (Lam Dong Hospital)

Non-Destructive Testing

Isotope Hydrology

Animal Science

Radiation Exposure Assessment

Workshop on Design, Management and Evaluation of TC Projects

Food Irradiation

Nuclear Analytical Services

Design and Production of Nuclear Instruments

Service Facilities for Radiation Detectors
Non-Destructive Testing
$0 \mathrm{~J}$

$5 \mathrm{~B}$

$6 \mathrm{~B}$

$8 C$

$8 \mathrm{C}$

\section{2-12-30 Aslam, Javed (TCPA)}

1992-12-30 Aslam, Javed (TCPA)

1992-12-30 Aslam, Javed (TCPA)

1992-12-30 Aslam, Javed (TCPA)

1992-12-30 Aslam, Javed (TCPA)

1993-08-26

Nemoto, Shinichiro Shane (TCPM)

1993-10-13 Nemoto, Shinichiro

Shane (TCPM)

$9 \mathrm{~K}$

1993-10-13 Nemoto, Shinichiro

Shane (TCPM)

OA 1993-10-18 Nemoto, Shinichiro

Shane (TCPM)

$81 \quad 1993-10-18 \quad$ Nemoto, Shinichiro

Shane (TCPM)

1994-01-28 Nemoto, Shinichiro

Shane (TCPM)

1994-05-25 Nemoto, Shinichiro

Shane (TCPM)

1995-08-02 Yang, Maeng-Ho
Akhtar, Khalid

Mahmud (NAPC)

Ahmed, Jasim Uddin

(NENS)

Vera Ruiz, Hernan

Guido (NAPC)

Taoka, Wesley M.

(NESI)

Kumarasinghe,

Kumarasinghege

Saliya (NAFA)

Gopinathan Nair,

Gopalan (NAHU)

Zatolokine, Boris

Vasilyevich (NAPC)

Zatolokine, Boris

Vasilyevich (NAPC)

Louvat, Didier (NSRW)

927778

Perera, Buthgamu

Mudiyanselage

Amithral Oswin

(NAFA)

Weiss, Bernard $\mathrm{H}$.

(NSNS)

Markovic, Vitomir M.

(NAPC)

Ahmed, Mainuddin

(NAFA)

Akhtar, Khalid

Mahmud (NAPC)

Walsh, Robin L.

(NAPC)

Kaufmann, Herbert

(NAPC)
20538

290288

58342

168228

109993

243314

178036

11896

21080

1360944

72685

145095

140316 


\begin{tabular}{|c|c|c|c|c|}
\hline VIE4012 & Nuclear Instrumentation Services & $4 G$ & $1995-08-02$ & $\begin{array}{l}\text { Yang, Maeng-Ho } \\
\text { (TCPM) }\end{array}$ \\
\hline VIE6017 & Development of Nuclear Medicine in South Vietnam & $6 \mathrm{~B}$ & $1995-08-02$ & $\begin{array}{l}\text { Yang, Maeng-Ho } \\
\text { (TCPM) }\end{array}$ \\
\hline VIE3003 & Uranium Ore Processing Laboratory & $3 F$ & $1995-11-30$ & $\begin{array}{l}\text { Yang, Maeng-Ho } \\
\text { (TCPM) }\end{array}$ \\
\hline VIE7002 & Analysis of Environmental Samples & $7 \mathrm{~J}$ & $1995-11-30$ & $\begin{array}{l}\text { Yang, Maeng-Ho } \\
\text { (TCPM) }\end{array}$ \\
\hline VIE3002 & Nuclear Analytical Techniques for Prospection & $\begin{array}{l}3 \mathrm{E} \\
8 \mathrm{E}\end{array}$ & $1995-12-14$ & $\begin{array}{l}\text { Yang, Maeng-Ho } \\
\text { (TCPM) }\end{array}$ \\
\hline VIE0006 & Manpower Development & $O A$ & $1995-12-22$ & $\begin{array}{l}\text { Yang, Maeng-Ho } \\
\text { (TCPM) }\end{array}$ \\
\hline VIE4010 & Renovation of the Dalat Reactor Control System & $4 G$ & $1995-12-22$ & $\begin{array}{l}\text { Yang, Maeng-Ho } \\
\text { (TCPM) }\end{array}$ \\
\hline VIE9004 & Radiation Protection Development & $9 \mathrm{C}$ & $1995-12-22$ & $\begin{array}{l}\text { Yang, Maeng-Ho } \\
\text { (TCPM) }\end{array}$ \\
\hline VIE6018 & $\begin{array}{l}\text { Upgrading Brachytherapy Services - Ho Chi Minh } \\
\text { City }\end{array}$ & $6 \mathrm{C}$ & $1996-12-23$ & $\begin{array}{l}\text { Kamel, Reyad Sherif } \\
\text { (TCAP) }\end{array}$ \\
\hline VIE7003 & Haematological Laboratory for Radiation Injury & $7 \mathrm{C}$ & $1996-12-23$ & $\begin{array}{l}\text { Kamel, Reyad Sherif } \\
\text { (TCAP) }\end{array}$ \\
\hline VIE4013 & Local Production of Nuclear Medical Instruments & $4 G$ & $1997-04-30$ & $\begin{array}{l}\text { Kamel, Reyad Sherif } \\
\text { (TCAP) }\end{array}$ \\
\hline VIE8009 & $\begin{array}{l}\text { NDT for Industrial \& Engineering Non-Metallic } \\
\text { Products }\end{array}$ & $8 \mathrm{C}$ & $1997-04-30$ & $\begin{array}{l}\text { Kamel, Reyad Sherif } \\
\text { (TCAP) }\end{array}$ \\
\hline VIE8007 & Establishment of Sedimentology Laboratory & $8 \mathrm{~N}$ & $1998-02-27$ & $\begin{array}{l}\text { Kamel, Reyad Sherif } \\
\text { (TCAP) }\end{array}$ \\
\hline VIE8011 & $\begin{array}{l}\text { Study of Sedimentation Rates Using Cs-137 and Pb- } \\
210\end{array}$ & $8 \mathrm{~N}$ & $1998-05-25$ & $\begin{array}{l}\text { Kamel, Reyad Sherif } \\
\text { (TCAP) }\end{array}$ \\
\hline VIE8008 & $\begin{array}{l}\text { Nuclear Techniques for Groundwater Pollution } \\
\text { Protection }\end{array}$ & $8 \mathrm{M}$ & $1998-08-28$ & $\begin{array}{l}\text { Kamel, Reyad Sherif } \\
\text { (TCAP) }\end{array}$ \\
\hline VIE6019 & $\begin{array}{l}\text { QA and QC Programme for Hospital Radiation } \\
\text { Facilities }\end{array}$ & $\begin{array}{l}6 \mathrm{C} \\
\mathrm{OQ}\end{array}$ & $2000-08-30$ & $\begin{array}{l}\text { Kamel, Reyad Sherif } \\
\text { (TCAP) }\end{array}$ \\
\hline VIE7004 & Establishment of a Tissue Bank & $7 \mathrm{E}$ & $2000-08-30$ & $\begin{array}{l}\text { Kamel, Reyad Sherif } \\
\text { (TCAP) }\end{array}$ \\
\hline
\end{tabular}

Kaufmann, Herbert (NAPC)

Yamasaki, Toshiro (NAHU)

Nicolet, Jean-Paul (NEFW)

Stone, Susan F. (NAHU)

Lewkowicz, Irene (NAPC)

Akhtar, Khalid Mahmud (NAPC)

Skornik, Karol (TCAF)

Matscheko, Georg (NAHU)

Levin, Victor Cecil (NA)

Mircheva, Jordanka (NAHU)

Kaufmann, Herbert (NAPC)

Dobrowolski, Marek Krzysztof (NAPC)

García Agudo, Edmundo (NAPC)

García Agudo, Edmundo (NAPC)

Groening, Manfred (NAPC)

Kamel, Reyad Sherif

Turai, Istvan (NSRW) Ortiz Lopez, Pedro (NSRW)

Kamel, Reyad Sherif

Tatsuzaki, Hideo

(NAHU)
87603

178213

134502

100004

282647

202935

352083

505606

206240

93338

120440

182261

196914

109521

202614

171977

128057 


\begin{tabular}{|c|c|}
\hline VIE5013 & $\begin{array}{l}\text { Improvement of Basic Food Crops Through Induced } \\
\text { Mutation }\end{array}$ \\
\hline VIE0009 & $\begin{array}{l}\text { Pre-Feasibility Study for the Introduction of Nuclear } \\
\text { Power }\end{array}$ \\
\hline VIE3004 & Rare Earth Extraction Technology \\
\hline VIE8010 & Radiation Sterilization of Health Care Products \\
\hline VIE9007 & $\begin{array}{l}\text { Infrastructure for Treatment \& Management of } \\
\text { Radwaste }\end{array}$ \\
\hline VIE2006 & $\begin{array}{l}\text { Nuclear Analysis of Food and Environmental } \\
\text { Samples }\end{array}$ \\
\hline VIE6020 & Strengthening Nuclear Medicine Services \\
\hline VIE8015 & $\begin{array}{l}\text { Strengthening the Capability of Radiotracers for } \\
\text { Enhancing Oil Recovery }\end{array}$ \\
\hline VIE2005 & $\begin{array}{l}\text { Automation and QA-QC in Neutron Activation } \\
\text { Analysis }\end{array}$ \\
\hline VIE2007 & $\begin{array}{l}\text { QA/QC of Mineral and Environmental Sample } \\
\text { Measurements Using Nuclear Analytical Techniques }\end{array}$ \\
\hline VIE6022 & Upgrading Nuclear Medicine Services \\
\hline VIE8012 & Ground Water and Nutrient Management \\
\hline VIE8013 & $\begin{array}{l}\text { Advanced NDT Methods for the Construction } \\
\text { Industry }\end{array}$ \\
\hline \multicolumn{2}{|c|}{ ancelled National Projects (2) } \\
\hline $\begin{array}{l}\text { Project } \\
\text { Number }\end{array}$ & Title \\
\hline VIE6014 & Nuclear Medicine Facilities \\
\hline
\end{tabular}

$5 \mathrm{C}$

2000-10-31

OE

30

$3 \mathrm{D}$

$8 \mathrm{E}$

$8 \mathrm{H}$

$9 \mathrm{E}$
$4 \mathrm{O}$

40

2C

$6 B$

$8 \mathrm{~J}$

$6 \mathrm{~B}$

$8 \mathrm{~A}$ 8C 2005-12-28 $\begin{aligned} & \text { Kamel, Reyad Sherif } \\ & \text { (TCAP) }\end{aligned}$

Field Cancelle
On

$6 B \quad 1988-10-19$ Aslam, Javed (TCPA)

2003-02-26 Kamel, Reyad Sherif (TCAP)

2003-03-28 Kamel, Reyad Sherif (TCAP)

2003-05-30 Kamel, Reyad Sherif (TCAP)

2004-08-27 Kamel, Reyad Sherif (TCAP)

2004-08-27 Kamel, Reyad Sherif (TCAP)

2005-12-28 Kamel, Reyad Sherif (TCAP)

Kamel, Reyad Sherif (TCAP)
Kamel, Reyad Sherif (TCAP)

Kamel, Reyad Sherif (TCAP)

Kamel, Reyad Sherif (TCAP)

Kamel, Reyad Sherif (TCAP)

Kamel, Reyad Sherif (TCAP)
Rossbach, Matthias $\mathrm{H}$. (NAPC)

Kamel, Reyad Sherif (TCAP)

Thereska, Jovan (NAPC)

Kamel, Reyad Sherif (TCAP)

Kamel, Reyad Sherif (TCAP)

Kamel, Reyad Sherif (TCAP)

Kamel, Reyad Sherif (TCAP)

Kamel, Reyad Sherif (TCAP)
Project Responsible

\section{Officer}

Nichterlein, Karin (NAFA)

Hamilton, Bruce (NE)

Rossbach, Matthias $\mathrm{H}$. (NAPC)

Gueven, Olgun (NAPC)

Al-Mughrabi, Mohamed (NEFW) Wieland, Patricia (NS)

Rossbach, Matthias $\mathrm{H}$. (NAPC)

Padhy, Ajit Kumar (NAHU)

Thereska, Jovan

(NAPC)

Rossbach, Matthias $\mathrm{H}$. (NAPC)

Rossbach, Matthias $\mathrm{H}$ (NAPC)

Dondi, Maurizio

(NAHU)

Groening, Manfred (NAPC)

Einav, Isaac (NAPC)

291073

147129

197218

528620

207751

269929

452814

173415

180989

174223

162237

225664

176905

\section{Technical Officer}

\section{Total Budget}




\subsection{Regional/Interregional Projects:}

\begin{tabular}{|c|c|c|c|c|}
\hline \multicolumn{5}{|c|}{ Active Regional/Interregional Projects (66) } \\
\hline $\begin{array}{l}\text { Project } \\
\text { Number }\end{array}$ & Title & Field & $\begin{array}{l}\text { 1st Year of } \\
\text { Approval }\end{array}$ & Country Officer \\
\hline INT0060 & $\begin{array}{l}\text { Technical Co-operation Between Developing } \\
\text { Countries }\end{array}$ & $O A$ & 1995 & $\begin{array}{l}\text { Salema, Manase Peter } \\
\text { (TCAP) }\end{array}$ \\
\hline INT4131 & $\begin{array}{l}\text { Sustainable Technologies for Managing Radioactive } \\
\text { Wastes }\end{array}$ & $3 \mathrm{H}$ & 1997 & $\begin{array}{l}\text { Kurghinyan, Vladimir } \\
\text { (TCEU) }\end{array}$ \\
\hline RAS6029 & $\begin{array}{l}\text { Distance-assisted Training for Nuclear Medicine } \\
\text { Technicians (RCA) }\end{array}$ & $6 \mathrm{~B}$ & 1997 & $\begin{array}{l}\text { Dias, Mahendra } \\
\text { Prinath (TCAP) }\end{array}$ \\
\hline INT5145 & $\begin{array}{l}\text { Insect Pest Control Using the Sterile Insect } \\
\text { Technique }\end{array}$ & $5 \mathrm{D}$ & 1999 & $\begin{array}{l}\text { Salema, Manase Peter } \\
\text { (TCAP) }\end{array}$ \\
\hline RAS5035 & $\begin{array}{l}\text { Improving Animal Productivity and Reproductive } \\
\text { Efficiency (RCA) }\end{array}$ & $5 \mathrm{E}$ & 1999 & $\begin{array}{l}\text { Dias, Mahendra } \\
\text { Prinath (TCAP) }\end{array}$ \\
\hline RAS6033 & Distance Education in Radiation Oncology (RCA) & $6 \mathrm{C}$ & 1999 & $\begin{array}{l}\text { Dias, Mahendra } \\
\text { Prinath (TCAP) }\end{array}$ \\
\hline RAS8084 & $\begin{array}{l}\text { Isotope Use in Managing and Protecting Drinking } \\
\text { Water (RCA) }\end{array}$ & $8 \mathrm{M}$ & 1999 & $\begin{array}{l}\text { Dias, Mahendra } \\
\text { Prinath (TCAP) }\end{array}$ \\
\hline RAS9023 & $\begin{array}{l}\text { Legislation for Safe and Peaceful Nuclear } \\
\text { Applications }\end{array}$ & $\begin{array}{l}0 D \\
9 C\end{array}$ & 1999 & Lutfi, Nabil (TCAP) \\
\hline
\end{tabular}

Project Responsible
Officer
Kurghinyan, Vladimir
(TCEU)
Dias, Mahendra
Prinath (TCAP)

Prinath (TCAP)
Lutfi, Nabil (TCAP)
Dias, Mahendra
Technical Officer
Salema, Manase Peter (TCAP)
Al-Mughrabi,
Mohamed (NEFW)
Balla, Janos (NEFW)
Kahraman, Alper

(NEFW)

Dondi, Maurizio

(NAHU)

Watanabe, Naoyuki

(NAHU)

Hendrichs, Jorge

Pedro (NAFA)

Boettcher, Paul John

(NAFA)

Vercoe, Philip Edward

(NAFA)

Hendry, Jolyon

Hindson (NAHU)

Kulkarni, Kshitij

Mahadev (NAPC)

Vez Carmona, Maria

de Lourdes (EXPO)

Djermouni, Belkacem (NSRW)

Total Budget

946144

2565864

636688

1936300

1052981

715200

589625

327847 
INT0074

Pre-Project Assistance (Africa and East Asia and the Pacific)

INT0076

INT0078 and the Pacific)

ICT-Assisted Training/Learning Materials in Nuclear Instrumentation Maintenance

RAS0032

Support Towards Self-Reliance and Sustainability of National Nuclear Institutions

RAS402

Management of Changes for Competitive Nuclear Power Performance

RAS5039

Restoration of Soil Fertility and Sustenance of Agricultural Productivity (RCA)

RAS5040 Enhancement of Genetic Diversity in Food, Pulses, and Oil Crops and Establishment of Mutant Germplasm Network (RCA)

RAS5041

Production of Foot and Mouth Disease Antigen and Antibody ELISA Reagent Kit (RCA)

RAS5042 Application of Food Irradiation for Food Security Safety, and Trade (RCA)

RAS6034 Quality Assurance Programme for Molecular-Based Diagnosis of Infectious Diseases

RAS8091

Process Diagnostics and Optimization in Petrochemical Industry (RCA)

RAS8092
Investigating Environment and Water Resources in Geothermal Areas (RCA)
Dias, Mahendra Prinath (TCAP)

$O A$
2001 Salema, Manase Peter (TCAP)

2001 Salema, Manase Peter (TCAP)

2001 Maksoudi, Mokdad (TCAF)

2001 Lutfi, Nabil (TCAP)

2001 Xu, Naicheng (TCAP)

2001 Dias, Mahendra Prinath (TCAP)

2001 Dias, Mahendra Prinath (TCAP) Kim, Myung Ro (TCAP)

2001 Dias, Mahendra Prinath (TCAP)

2001 Dias, Mahendra Prinath (TCAP)

2001 Kamel, Reyad Sherif (TCAP)

2001 Dias, Mahendra Prinath (TCAP)

2001 Dias, Mahendra Prinath (TCAP)
Dias, Mahendra

Prinath (TCAP)

Telleria, Diego Miguel

(NSRW)

Shakhashiro,

Abdulghani (NAAL)

Salema, Manase Peter Salema, Manase Peter (TCAP)

(TCAP)

Salema, Manase Peter (TCAP)

Maksoudi, Mokdad (TCAF)

Salema, Manase Peter (TCAP)

Markowicz, Andrzej

(NAAL)

Kaufmann, Herbert (NAPC)

Lutfi, Nabil (TCAP)

Dias, Mahendra Prinath (TCAP) Lutfi, Nabil (TCAP)

Xu, Naicheng (TCAP)

Mazour, Thomas Joseph (NENP) Kang, Ki-Sig Sig (NENP)

Zapata, Felipe (NAFA)

Shu, Qingyao (NAFA) Lokko, Yvonne

Rosaline Naa (NAFA)

Lagoda, Pierre Jean Laurent (NAFA)

Crowther, John Richard (NAFA)

Rubio Cabello, Wilma Tatiana (NAFA)

Kamel, Reyad Sherif

Khan, Baldip Kaur (NAHU)

\section{(TCAP)}

Jin, Joon-Ha (NAPC)

Dias, Mahendra

Prinath (TCAP)

Einav, Isaac (NAPC)

Dias, Mahendra

Kulkarni, Kshitij

Mahadev (NAPC)
138708

539136

109200

357650

660166

1036304

619203

607540

191210

226263

863110

723375

287826 
RAS8093 Use of Isotopes in Dam Safety and Dam Sustainability (RCA)

RAS9025

RAS9026

RAS9027

Development of Technical Capabilities fo Sustainable Radiation and Waste Safety Infrastructure

mproving the Quality of Production and Uses of Radiation Sterilized Tissue Grafts

INT4141 Status and Prospects of Development for and Applications of Innovative Reactor Concepts for Developing Countries

INT5147

Developing Salt-tolerant Crops for Sustainable Food and Feed Production in Saline Lands

INT5148

Establishing Quality Systems in Veterinary Testing Laboratories

Management of Technical Cooperation among Developing Countries (RCA)
2001 Dey, Sujit (TCAP)

Dey, Sujit (TCAP)

2001 Dias, Mahendra Prinath (TCAP)

2001 Xu, Naicheng (TCAP)

Xu, Naicheng (TCAP)

Dey, Sujit (TCAP)

2001 Dey, Sujit (TCAP)

ey, Sujit (TCAP)

2002 Salema, Manase Peter (TCAP)

2003 Cardoso Cabezon, Alain Jorge (TCEU)

003 Salema, Manase Peter (TCAP)

2003 Salema, Manase Peter (TCAP)

2003 Dias, Mahendra Prinath (TCAP)

Cardoso Cabezon, (TCAP)

Dias, Mahendra Prinath (TCAP)

2003 Salema, Manase Peter

Dias, Mahendra
Prinath (TCAP)
Xu, Naicheng (TCAP)
Dey, Sujit (TCAP)

Araguas Araguas, Luis Jesus (NAPC)

Kurttas, Tuerke (NAPC)

Nichols, Robert Charles (NSNI)

Mrabit, Khammar (NSRW)

O'Donnell Torroba Patricio Alfonso (NSRW)

\section{Mrabit, Khammar} (NSRW)

O'Donnell Torroba,

Patricio Alfonso

(NSRW)

Hendry, Jolyon

Hindson (NAHU)

Hindson (NAHU)

Kuznetsov, Vladimir (NENP)

Salema, Manase Peter

Spencer, Marie Madeleine (NAFA) Miranda, Manoela Pessoa de (NAFA)

Lagoda, Pierre Jean Laurent (NAFA)

Viljoen, Gerrit

Johannes (NAFA)

Crowther, John

Richard (NAFA)

Dias, Mahendra Prinath (TCAP) (TCAP)
Salema, Manase Peter (TCAP)
Salema, Manase Peter (TCAP)
307631

717295

1719895

2277735

844860

883991

336300

229580

997867

481900 
RAS0037

Support to Member States on Design and Formulation of Project Proposals

RAS0038

RAS4022

RAS4023

RAS6036

RAS6037

RAS6038

RAS6039

RAS7011

RAS7013

Improved Information about Urban Air Quality Management (RCA)

RAS7014

Monitoring of Food Fortification Programmes Using Nuclear Techniques

Optimization of Materials in Industry Using Online Bulk Analysis Techniques (RCA) Competitive Electricity Markets (RCA)

Improvement of Research Reactor Operation and

Repair and Refurbishment of Nuclear Instruments

Management of Liver Cancer Using Transarterial Radioconjugate Therapy (RCA) by Radiotherapy (RCA) and Training (RCA)

Use of Radiosynovectomy in the Management of Patients Suffering from Painful Joint Disorders (RCA)

Enhancing the Sustainability of the Marine Coastal Environment (RCA)
$6 \mathrm{~J}$

2003 Salema, Manase Peter (TCAP)

2003 Dias, Mahendra Prinath (TCAP)

2003 Dias, Mahendra Prinath (TCAP)

2003 Lutfi, Nabil (TCAP)

2003 Dias, Mahendra Prinath (TCAP)

\section{Dias, Mahendra} Prinath (TCAP)

2003 Dias, Mahendra Prinath (TCAP)

2003 Dias, Mahendra Prinath (TCAP)

2003 Dias, Mahendra Prinath (TCAP) Prinath (TCAP)

2003 Sukasam, Kesrat (TCAP)

2003 Dias, Mahendra Prinath (TCAP)
Salema, Manase Peter (TCAP)

Dias, Mahendra

Prinath (TCAP)

Dias, Mahendra

Prinath (TCAP)

Lutfi, Nabil (TCAP)

Dias, Mahendra

Prinath (TCAP)

Dias, Mahendra

Prinath (TCAP)

Dias, Mahendra

Prinath (TCAP)

Dias, Mahendra

Prinath (TCAP)

Dias, Mahendra

Prinath (TCAP)

Dias, Mahendra Prinath (TCAP)

Sukasam, Kesra (TCAP) (NAPC)

(NAHU)

Watanabe, Naoyuki

(NAHU)

(NAHU)

Dondi, Maurizio

Watanabe, Naoyuki

(NAHU)

Zaknun, John (NAHU)

(NAML)

(NAML)

(NAAL)

Lagoda, Pierre Jean

Palad (NAHU)

Dias, Mahendra Prinath (TCAP)
Salema, Manase Peter (TCAP)

Jalal, Ahmed Irej (NE)

Paranjpe, Shriniwas Krishnarao (NAPC)

Kaufmann, Herbert

Dondi, Maurizio

(NAHU)

Rosenblatt, Eduardo

McLean, Ian Donald

(NAHU)

Boisson, Florence

Jeffree, Ross A.

Trinidad, Trinidad

Palad (NAHU)

Markowicz, Andrzej

Laurent (NAFA)

Trinidad, Trinidad

293667

228735

209975

316670

217210

212000

477264

169463

305888

810946

Jin, Joon-Ha (NAPC) 

Emergencies (RCA)

RAS8096

Modification of Natural Polymers through Radiation Processing (RCA)

RAS8097

RAS9028

RAS9029

INT5149

RAS0041

RAS4024

RAS5043

RAS5044

RAS6040

mprovement in Quality of Radiotherapy for Frequen Cancers in the Region (RCA)

RAS6041

sotope Techniques for Groundwater Contamination Studies in Urbanized and Industrial Areas (RCA)

Development of an Asian Nuclear Safety Network

Harmonization of Radiation Protection, Phase IV

(RCA)

Training Course on the Use of the Sterile Insect and Related Techniques

Tracing Future Sustainable Paths through Nuclear and Other Energy Options (RCA)

Radioisotope Production and Neutron Beam Applications (RCA)

Sustainable Land Use and Management Strategies for Controlling Soil Erosion and Improving Soil and Water Quality (RCA)

ntegrated Approach for Improving Livestock

Production Using Indigenous Resources and

Conserving the Environment (RCA)
Dias, Mahendra

Prinath (TCAP)

Prinath (TCAP)

2003 Dias, Mahendra Prinath (TCAP)

Dias, Mahendra

Prinath (TCAP)

2003 Dias, Mahendra Prinath (TCAP)

2003 Lutfi, Nabil (TCAP)

2003 Dias, Mahendra Prinath (TCAP)

2005 Boussaha, Ali (TCAF)

2005 Dias, Mahendra Prinath (TCAP)

2005 Dias, Mahendra Prinath (TCAP)

2005 Dias, Mahendra Prinath (TCAP) Kim, Myung Ro (TCAP)

2005 Dias, Mahendra Prinath (TCAP) Kim, Myung Ro (TCAP)

2005 Dias, Mahendra Prinath (TCAP)

2005 Dias, Mahendra Prinath (TCAP)
Dias, Mahendra

Prinath (TCAP)

Lutfi, Nabil (TCAP)

Dias, Mahendra

Prinath (TCAP)

Boussaha, Ali (TCAF)

Dias, Mahendra Prinath (TCAP)

Dias, Mahendra Prinath (TCAP)

Dias, Mahendra Prinath (TCAP)

Dias, Mahendra Prinath (TCAP)
Scholten, Jan

Christoph (NAML)

Sampa, Maria Helena

de O. (NAPC)

Kulkarni, Kshitij

Mahadev (NAPC)

Lederman, Luis (NS)

O'Donnell Torroba,

Patricio Alfonso

(NSRW)

Mrabit, Khammar

(NSRW)

Hendrichs, Jorge

Pedro (NAFA)

Jalal, Ahmed Irej (NE)

Paranjpe, Shriniwas Krishnarao (NAPC)

Pillai, Maroor

Raghavan

Ambikalmajan (NAPC)

Fulajtar, Emil (NAFA)

Boettcher, Paul John

(NAFA)

Vercoe, Philip Edward

(NAFA)

Rosenblatt, Eduardo

(NAHU)

McLean, Ian Donald

(NAHU)

Davidsson, Lena

Margareta (NAHU)
227440

370400

125911

462337

219490

779884

335763

893346

398180 


\begin{tabular}{|c|c|}
\hline RAS6042 & Tumour Imaging Using Radioisotopes (RCA) \\
\hline RAS6043 & $\begin{array}{l}\text { Regional Screening Network for Neonatal } \\
\text { Hypothyroidism, Phase II }\end{array}$ \\
\hline RAS8098 & $\begin{array}{l}\text { Radiation Technology for Development of Advanced } \\
\text { Materials and for Protection of Health and the } \\
\text { Environment (RCA) }\end{array}$ \\
\hline RAS8099 & $\begin{array}{l}\text { Radioisotope Technology for Natural Resource } \\
\text { Exploration and Exploitation (RCA) }\end{array}$ \\
\hline RAS8100 & Advanced Industrial Radiography (RCA) \\
\hline RAS9033 & $\begin{array}{l}\text { Developing Technical Capabilities for the Protection } \\
\text { of Health and Safety of Workers Exposed to lonizing } \\
\text { Radiation }\end{array}$ \\
\hline
\end{tabular}

RAS9034

RAS9035

RAS9036
Establishment of National Capabilities for Response to a Radiological and Nuclear Emergency
2005 Dias, Mahendra Prinath (TCAP)

2005 Kamel, Reyad Sherif (TCAP)

2005 Dias, Mahendra Prinath (TCAP)

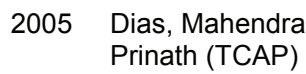

2005 Dias, Mahendra Prinath (TCAP)

2005 Dey, Sujit (TCAP)

2005 Dey, Sujit (TCAP)

2005 Dey, Sujit (TCAP)

2005 Dey, Sujit (TCAP)
Dias, Mahendra

Prinath (TCAP)

Kamel, Reyad Sherif (TCAP)

Dias, Mahendra

Prinath (TCAP)

Dias, Mahendra

Prinath (TCAP)

Dias, Mahendra

Prinath (TCAP)

Dey, Sujit (TCAP)
Watanabe, Naoyuki (NAHU)

Solanki, Kishor K. (NAHU)

Sampa, Maria Helena de O. (NAPC)

Jin, Joon-Ha (NAPC)

Einav, Isaac (NAPC)

Deboodt, Pascal P. A. (NSRW)

Patricio Alfonso

(NSRW)

Mrabit, Khammar (NSRW)

Rehani, Madan Mohan (NSRW)

O'Donnell Torroba,

Patricio Alfonso

(NSRW)

Mrabit, Khammar

(NSRW)

Zombori, Peter (NS

O'Donnell Torroba,

Patricio Alfonso

(NSRW)

Djermouni, Belkacem

(NSRW)

O'Donnell Torroba,

Patricio Alfonso

(NSRW)

Mrabit, Khammar

(NSRW)
323350

243530

197650

400020

409070

275560

338993

251900

674600 


\section{Field}

$\begin{array}{ll}\begin{array}{l}\text { Project } \\ \text { Number }\end{array} & \text { Title } \\ \text { RAS5020 } & \begin{array}{l}\text { Food Irradiation Process Control and Acceptance } \\ \text { (RCA) }\end{array}\end{array}$

INT9144

Upgrading Waste Management Infrastructure

RAS8071

Isotopes \& Rad. for Tech. \& Environ. Sustainable Devp. (RCA)

RAS502

Increasing the Capabilities of Common Grain Legumes (RCA)

RAS7003 Radiation Sterilization of Tissue Grafts (RCA)

RAS4011 Research Reactor Utilization (RCA)

RAS6022

RAS8068

\section{Strengthening Nuclear Medicine in RCA Member} States (RCA)

Isotopes and Radiation in Industry and Environment (RCA)

$\begin{array}{ll}\begin{array}{l}\text { Completed } \\ \text { on }\end{array} & \begin{array}{l}\text { Country Officer } \\ 1996-05-13\end{array} \\ \begin{array}{l}\text { Easey, John Frederick } \\ \text { (TCPM) }\end{array} \\ 1997-09-27 & \begin{array}{l}\text { Barretto de Campos, } \\ \text { Paulo Marcos (TCPB) }\end{array} \\ 1998-04-17 & \begin{array}{l}\text { Aleta, Carlito Ronguillo } \\ \text { (TCPA) }\end{array}\end{array}$

1998-05-0

Aleta, Carlito Ronguillo (TCPA)

7E 1998-08-28 Aleta, Carlito Ronguillo (TCPA)

4B 1998-08-31 Aleta, Carlito Ronguillo (TCPA)

6B 1998-08-31 Aleta, Carlito Ronguillo (TCPA)

$8 \mathrm{C}$
Dey, Sujit (TCAP)

(NSRW)

\begin{tabular}{|c|c|c|}
\hline \multirow{9}{*}{$\begin{array}{l}\text { Project Responsible } \\
\text { Officer }\end{array}$} & Technical Officer & Total Budget \\
\hline & $\begin{array}{l}\text { Loaharanu, Paisan } \\
\text { (NAFA) }\end{array}$ & 523847 \\
\hline & Bergman, Curt (NEPF) & 174031 \\
\hline & $\begin{array}{l}\text { Thereska, Jovan } \\
\text { (NAPC) } \\
\text { Gueven, Olgun } \\
\text { (NAPC) } \\
\text { Walsh, Michael A.R. } \\
\text { (NAPC) }\end{array}$ & 2549371 \\
\hline & Serraj, Rachid (NAFA) & 918530 \\
\hline & $\begin{array}{l}\text { Tatsuzaki, Hideo } \\
\text { (NAHU) }\end{array}$ & 1050580 \\
\hline & Dimic, Viktor (NAPC) & 368146 \\
\hline & $\begin{array}{l}\text { Padhy, Ajit Kumar } \\
\text { (NAHU) }\end{array}$ & 326653 \\
\hline & Thereska, Jovan & 780874 \\
\hline
\end{tabular}

Sadagopan, Geetha (NSRW)

O'Donnell Torroba,

Patricio Alfonso

(NSRW)

Mrabit, Khammar

Adelfang, Pablo

(NEFW)

8949388

992933

(NAPC) 


\begin{tabular}{|c|c|}
\hline RAS8070 & $\begin{array}{l}\text { Isotopes and Radiation in Industry and the } \\
\text { Environment (RCA) }\end{array}$ \\
\hline RAS0022 & $\begin{array}{l}\text { Public Acceptance and Trade in Irradiated Food } \\
\text { (RCA) }\end{array}$ \\
\hline RAS6018 & Radioimmunoassay for Hepatitis B Diagnosis (RCA) \\
\hline RAS0021 & Nuclear Power Planning (RCA) \\
\hline RAS0023 & $\begin{array}{l}\text { Energy, Electricity and Nuclear Power Planning } \\
\text { (RCA) }\end{array}$ \\
\hline RAS9006 & $\begin{array}{l}\text { Strengthening of Radiation Protection Infrastructures } \\
\text { (RCA) }\end{array}$ \\
\hline INT9143 & Upgrading Radiation Protection Infrastructure \\
\hline RAS0024 & Project Formulation Meetings (RCA) \\
\hline RAS5034 & $\begin{array}{l}\text { Irradiation As Sanitary \& Phytosanitary Food } \\
\text { Treatment (RCA) }\end{array}$ \\
\hline RAS4019 & $\begin{array}{l}\text { Improving Research Reactor Operation and } \\
\text { Utilization (RCA) }\end{array}$ \\
\hline RAS8078 & $\begin{array}{l}\text { Nucleonic Control Systems and Tracers in Industry } \\
\text { (RCA) }\end{array}$ \\
\hline RAS8080 & $\begin{array}{l}\text { Better Mgt of Envir \& Industry Thru Isotope \& Rad } \\
\text { Tech (RCA) }\end{array}$ \\
\hline RAS8077 & $\begin{array}{l}\text { Thematic Programme on Advanced Techniques for } \\
\text { Industry (RCA) }\end{array}$ \\
\hline RAS0028 & $\begin{array}{l}\text { Comparative Assessmt of Electricity Generation } \\
\text { Options (RCA) }\end{array}$ \\
\hline
\end{tabular}

\begin{tabular}{|c|c|c|}
\hline $\begin{array}{l}8 \mathrm{C} \\
8 \mathrm{H}\end{array}$ & $1998-08-31$ & $\begin{array}{l}\text { Aleta, Carlito Ronguillo } \\
\text { (TCPA) }\end{array}$ \\
\hline $\begin{array}{c}8 \mathrm{G} \\
0 \mathrm{~K} \\
5 \mathrm{H}\end{array}$ & $1998-12-11$ & $\begin{array}{l}\text { Aleta, Carlito Ronguillo } \\
\text { (TCPA) }\end{array}$ \\
\hline $6 B$ & $1998-12-23$ & $\begin{array}{l}\text { Aleta, Carlito Ronguillo } \\
\text { (TCPA) }\end{array}$ \\
\hline $\begin{array}{l}O A \\
O B\end{array}$ & $1999-11-30$ & $\begin{array}{l}\text { Aleta, Carlito Ronguillo } \\
\text { (TCPA) }\end{array}$ \\
\hline $\begin{array}{l}0 A \\
0 B \\
0 J\end{array}$ & $1999-12-21$ & $\begin{array}{l}\text { Aleta, Carlito Ronguillo } \\
\text { (TCPA) }\end{array}$ \\
\hline $9 \mathrm{C}$ & $1999-12-21$ & $\begin{array}{l}\text { Aleta, Carlito Ronguillo } \\
\text { (TCPA) }\end{array}$ \\
\hline
\end{tabular}

2001-05-31

$5 \mathrm{H}$

Barretto de Campos, Paulo Marcos (TCPB) (TCPA) (TCPA) (TCPA)

2002-05-31 Aleta, Carlito Ronguillo (TCPA) (TCPA)

2002-07-30 Aleta, Carlito Ronguillo (TCPA)
Thereska, Jovan (NAPC)

Loaharanu, Paisan (NAFA)

Ch'Ng, Soo Ling (NAHU)

Mazour, Thomas Joseph (NENP)

Mazour, Thomas

Joseph (NENP)

Hamilton, Bruce (NE)

Oresegun, Modupe

Olusola (NSRW)

Mrabit, Khammar

(NSRW)

Barretto de Campos,

Paulo Marcos (TCPB)

Gonzalez, Abel Julio (NSRW)

Mrabit, Khammar (NSRW)

Aleta, Carlito Ronguillo Aleta, Carlito Ronguillo

(TCPA)

Aleta, Carlito Ronguillo (TCPA)

2001-08-27 Aleta, Carlito Ronguillo Aleta, Carlito Ronguillo (TCPA)

Loaharanu, Paisan (NAFA)

2001-09-13 Aleta, Carlito Ronguillo Aleta, Carlito Ronguillo (TCPA)

Aleta, Carlito Ronguillo (TCPA)

Basu, Saibal (NAPC)

Thereska, Jovan (NAPC)

2002-07-15 Aleta, Carlito Ronguillo Aleta, Carlito Ronguillo (TCPA)

Turner, Jeffrey Vaughan Vaughan (NAPC)

2003-01-30 Aleta, Carlito Ronguillo

Aleta, Carlito Ronguillo (TCPA)

Einav, Isaac (NAPC)

Bui, Duy Thanh (NE) (TCPA)
1006110

86048

922575

165258

410427

1597518

5306472

384759

175852

192778

391643

811067

364835

436979 


$\begin{array}{ll}\text { RAS4016 } & \begin{array}{l}\text { Disposal of Radioactive Waste from Non-Power } \\ \text { Sources (RCA) }\end{array} \\ \text { RAS8089 } & \begin{array}{l}\text { Optimization of Mineral Resources Recovery Using } \\ \text { Low Radioactivity Portable Nucleonic Gauges (RCA) }\end{array} \\ \text { RAS0033 } & \begin{array}{l}\text { Role of Nuclear Power and Other Energy Options in } \\ \text { Mitigating Greenhouse Gas Emissions (RCA) }\end{array} \\ \text { RAS4020 } & \begin{array}{l}\text { Improvement of Research Reactor Operation and } \\ \text { Utilization (RCA) }\end{array} \\ \text { RAS8083 } & \begin{array}{l}\text { Management of Marine Coastal Environmental } \\ \text { Pollution (RCA) }\end{array}\end{array}$

RAS8086

RAS9021

RAS7008

RAS5029 Nuclear Techniques for the Promotion of Agroforestry

RAS0030

RAS7010

RAS0025

RAS5037
Quality Assurance in Radiation Sterilization of Tissue Graft (RCA) Systems

$7 \mathrm{E}$

\section{$5 B$}

$7 \mathrm{~J}$

Measuring the Effectiveness of Multinutrient Supplementation

Technical Co-operation Among Developing Countries (RCA)

Mutational Enhancement for Genetic Diversity in Rice (RCA)

\section{0}

$8 \mathrm{C}$

OE

4B

$8 \mathrm{G}$

$8 G$

2003-02-26

Radiotracers/Sealed Sources/Nucleonic Gauges in Indust.(RCA)

Upgrading Radiation Protection Infrastructure
$8 J$
2003-02-26

2003-03-28 Aleta, Carlito Ronguillo
(TCPA)

Dey, Sujit (TCAP)
Aleta, Carlito Ronguillo (TCPA)

Aleta, Carlito Ronguillo (TCPA)

Aleta, Carlito Ronguillo (TCPA)

Aleta, Carlito Ronguillo (TCPA)

Aleta, Carlito Ronguillo (TCPA)
2003-04-29

2003-05-30

$2003-06-25$

2003-12-31

2004-04-30

2004-04-30
Aleta, Carlito Ronguillo (TCPA)

Lutfi, Nabil (TCAP)

Lutfi, Nabil (TCAP)

Lutfi, Nabil (TCAP)

Dias, Mahendra Prinath (TCAP)

Dias, Mahendra Prinath (TCAP)
Kim, Kyoung-Pyo (TCPA)

Aleta, Carlito Ronguillo (TCPA)

Kim, Kyoung-Pyo (TCPA)

Kim, Kyoung-Pyo (TCPA)

(TCPA)
Aleta, Carlito Ronguillo (TCPA)

Dey, Sujit (TCAP)
Aleta, Carlito Ronguillo (TCPA)

Lutfi, Nabil (TCAP)

Lutfi, Nabil (TCAP)

Lutfi, Nabil (TCAP)

Dias, Mahendra

Prinath (TCAP)

Kim, Kyoung-Pyo (TCPA)
Dayal, Ramesh

(NEFW)

Thereska, Jovan (NAPC)

Bui, Duy Thanh (NE)

Paranjpe, Shriniwas Krishnarao (NAPC)

Fowler, Scott W.

(NAML)

Boisson, Florence

(NAML)

Turner, Jeffrey

Vaughan Vaughan

(NAPC)

Thereska, Jovan (NAPC)

Mrabit, Khammar (NSRW)

Bilbao Alfonso,

Alejandro Victor

(NSRW)

Hendry, Jolyon Hindson (NAHU)

Keerthisinghe

Deegala Gamini

(NAFA)

156601

312352

202399

238067

201587

532451

2418788

Razley, Mohd. Nordin

(TCPA)

Iyengar, Govindaraja Venkatesha (NAHU)

Dias, Mahendra

Prinath (TCAP)

Shu, Qingyao (NAFA)

920506

390965

667493

475399

490260

335110 

RAS6035 LDR and HDR Brachytherapy in Treating Cervical
Cancer (RCA) Conservation Through Radiation Processing (RCA)

RAS0029 Radiation Protection and Networking (RCA)

2004-05-20

Quality Assurance and Good Manufacturing Practice for Radiopharmaceuticals

RAS4017

Maintenance and Repair of Nuclear Instruments

Nuclear Medicine Applications (RCA)

RAS6028

RAS6027
2004-05-20

2004-05-20

2004-05-20
Dias, Mahendra Prinath (TCAP)

Dias, Mahendra Prinath (TCAP)

Dias, Mahendra Prinath (TCAP)

Lutfi, Nabil (TCAP)

Lutfi, Nabil (TCAP)

Lutfi, Nabil (TCAP)

Dias, Mahendra Prinath (TCAP) Prinath (TCAP) Prinath (TCAP)

Dias, Mahendra Prinath (TCAP)
Vikram, Bhadrasain

(NA)

Shortt, Kenneth Rober

(NAHU)

Pernicka, Frantisek

(NAHU)

Chmielewski, Andrzej

Grzegorz (NAPC)

Sadagopan, Geetha (NSRW)

Pillai, Maroor

Raghavan

Ambikalmajan (NAPC)

Kaufmann, Herbert

(NAPC)

Padhy, Ajit Kumar

(NAHU)

Solanki, Kishor K.

(NAHU)
Vikram, Bhadrasain

(NA)

Shortt, Kenneth Robert (NAHU)

Pernicka, Frantisek

(NAHU)
241489

75359

176735

322754

596355

1932912

774115 
Isotopic and Related Techniques to Assess Air Pollution - Joint UNDP/RCA (RCA)

Harmonization of Radiation Protection (RCA)

RAS9018

RAS7012

Diagnosing Osteoporosis Using Nuclear Techniques (RCA)

RAS2010

Quality Assurance and Quality Control of Nuclear

Analytical Techniques

RAS8085

RAS6032 Regional Screening Network for Neonata

Hypothyroidism

\section{Cancelled Regional/Interregional Projects (2)}

Project Title

RAS5036

Monitoring Pesticide Residues in Food \& the Environment(RCA)

Dias, Mahendra

Prinath (TCAP)
7J

$2004-12-30$

Dias, Mahendra

Prinath (TCAP)

$2 \mathrm{C}$

2005-02-28

Lutfi, Nabil (TCAP)

$8 \mathrm{C}$

2005-06-30

Dias, Mahendra Prinath (TCAP)

Kamel, Reyad Sherif (TCAP)

$\begin{array}{cll}\text { Field } & \begin{array}{l}\text { Cancelled } \\ \text { On }\end{array} & \begin{array}{l}\text { Country Officer } \\ \text { 5G }\end{array} \\ 2000-12-13 & \begin{array}{l}\text { Aleta, Carlito Ronguillo } \\ \text { (TCPA) }\end{array}\end{array}$

6B 2005-12-28
Dias, Mahendra
Prinath (TCAP)

Yonezawa, Chushiro

(NAHU)

Boisson, Florence

(NAML)

Markowicz, Andrzej

(NAAL)

Pang, Zhonghe

(NAPC)

Kemppainen, Markku

(TCPC)

Chmielewski, Andrze

Grzegorz (NAPC)

Jin, Joon-Ha (NAPC)

Markowicz, Andrzej

(NAAL)

Dias, Mahendra

Prinath (TCAP)

Yonezawa, Chushiro

(NAHU)

Dias, Mahendra

Prinath (TCAP)

Djermouni, Belkacem

(NSRW)

Mrabit, Khammar

(NSRW)

1045640

Mokhtar, Najat

(NAHU)

Prinath (TCAP)

Lutfi, Nabil (TCAP)

Rossbach, Matthias $\mathrm{H}$.

(NAPC)

Dias, Mahendra

Prinath (TCAP)

Einav, Isaac (NAPC)

Solanki, Kishor K

(NAHU)

$\begin{array}{ll}\begin{array}{l}\text { Project Responsible } \\ \text { Officer }\end{array} & \text { Technical Officer } \\ & \begin{array}{l}\text { Ferris, lan Glen } \\ \text { (NAFA) }\end{array}\end{array}$

\section{Total Budget}

(TCAP)
573112

1805756

29106

420013

229418

619108 
1 “IAEA National Training Course on 'Techniques and Strategies for Rice Quality and Nutrition Improvement,”” 7-12 August, 2006, http://wwwnaweb.iaea.org $/$ nafa/pbg/public/pb-nl-17.pdfAsearch $=\% 22$ vietnam $\% 22$

2 "Villages of Hope: The Ricemakers of Vietnam,"IAEA News Centre Features, 18 October 2005,

http://www.iaea.org/NewsCenter/Features/Vietnam/villages_of_hope.html\#

3 “The Nuclear Power Option in Vietnam," Traan Hu Phat, The International Journal of Nuclear Power, vol. 13 no. 4.,

http://www.npcil.nic.in/nupower_vol13_4/npovavol4.htm

4 “Vietnam," IAEA Publications, p. 914, 2002, http://www-

pub.iaea.org/MTCD/publications/PDF/cnpp2003/CNPP_Webpage/PDF/2002/Documents/Documents/Viet\%20Nam\%202002.pdf\#search=\%22vietnam\%22

${ }_{5}^{5}$ CIA World Factbook: Vietnam, 22 August 2006, https://www.cia.gov/cia/publications/factbook/geos/vm.html

${ }^{6}$ Wikipedia: Economy of Vietnam, http://en.wikipedia.org/wiki/Economy of Vietnam

7 "Vietnam, France Sign Nuclear Power Deal," Asia Times Online, 27 May 2004, http://www.nuclearpolicy.org/index.cfm/Page/Article/ID/1616

8 "The Nuclear Power Option in Vietnam," Traan Hu Phat, The International Journal of Nuclear Power, vol. 13 no. 4.

http://www.npcil.nic.in/nupower_vol13 4/npovavol4.htm

9 “Nuclear Power Among Non-fossil Options,"Inter Press Service News Agency, 31 August 2006, http://ipsnews.net/news.asp?idnews=32466

10 "Status of Dalat Research Reactor and Progress of New Reactor Plan in Vietnam," Nguyen Nhi Dien and Luong Ba Vien, Nuclear Research Institute, http://hanaro2005.kaeri.re.kr/data/proceeding/RR-O-04.pdftsearch=\%22dalat\%20reactor\%22

${ }^{11}$ Vietnam Country Plan, International Radiological Threat Reduction Program, FY06 Revision 0 (10/1/2006)

12 "Status of Dalat Research Reactor and Progress of New Reactor Plan in Vietnam," Nguyen Nhi Dien and Luong Ba Vien, Nuclear Research Institute, http://hanaro2005.kaeri.re.kr/data/proceeding/RR-O-04.pdf\#search=\%22dalat\%20reactor\%22

${ }_{14}^{13}$ Vietnam Country Plan, International Radiological Threat Reduction Program, FY06 Revision 0 (10/1/2006)

${ }^{14}$ Ibid.

${ }^{15}$ Ibid.

16 "Application of Knowledge Management on Nuclear Safety (Education and Training)," Presentation to Korean Atomic Energy Research Institute, 9-14 November 2003, http://www-ns.iaea.org/downloads/coordination/KM/Daejon/Files/1111/Country\%20Reports/Vietnam.ppt

17 "Russia Offers to Help Build Vietnam's First Nuclear Power Plant," RadioFreeEurope, 16 February 2006, http://www.globalsecurity.org/wmd/library/news/russia/2006/russia-060216-rferl02.htm

18 "Vietnam to Build First Nuclear Power Plant," China View, 19 October 2005, http://news.xinhuanet.com/english/2005-10/19/content 3648626.htm

19 "Experts fret over Human Shortage for Vietnam Nuclear Plant," Thanh Nien News.com, 29 August 2006, http://www.thanhniennews.com/politics/?catid=1\&newsid=19444

20 "Signing Training Agreement on Nuclear Power Technology between Hanoi University of Technology, Toshiba Company and Vietnam Atomic Energy Commission," 25 August 2006, http://www.vaec.gov.vn/News/Index.php?EV=0

21 "Vietnam News," Nuclear.com, 20 October 2005, http://www.nuclear.com/nation-by-nation/Vietnam_news.html 
22 “Application of Knowledge Management on Nuclear Safety (Education and Training),” Presentation to Korean Atomic Energy Research Institute, 9-14 November 2003, http://www-ns.iaea.org/downloads/coordination/KM/Daejon/Files/1111/Country\%20Reports/Vietnam.ppt

${ }^{23}$ Vietnam Atomic Energy Commission website, http://www.rca.iaea.org/regional/newFiles/vie.html

24 "Radiation and Nuclear Regulatory Structure in Vietnam," Vietnam Agency for Radiation and Nuclear Safety \& Control, November 2005, p. 1, 5.

${ }^{25}$ Ibid., pp. 1-5.

${ }^{26}$ Ibid.

${ }^{27}$ IAEA Technical Cooperation Country Profiles: Vietnam, 28 August 2006, http://www-tc.iaea.org/tcweb/default.asp 